\title{
Psoriasis in special localizations
}

\section{Klaudia Dopytalska, Piotr Sobolewski, Agata Błaszczak, Elżbieta Szymańska, Irena Walecka}

Department of Dermatology, Centre of Postgraduate Medical Education, Central Clinical Hospital of the Ministry of the Interior, Warsaw, Poland

\begin{abstract}
Psoriasis is a chronic inflammatory dermatosis affecting 1-3\% of the general population. Patients with psoriasis represent a heterogeneous population with individual disease expression - different degrees and severity of skin involvement. Psoriatic lesions in particular localizations such as the face, scalp, intertriginous or palmoplantar areas significantly reduce quality of life. Patients often feel ashamed, embarrassed, or self-conscious about their symptoms. Furthermore, genital psoriasis significantly affects sexual health. Among patients with psoriasis, the prevalence of special localizations is estimated to be $23-27 \%$ on the nails, $49 \%$ on the face, $12-16 \%$ on the palms and soles, and up to $36 \%$ in intertriginous regions. Due to peculiar features of skin in these areas, adequate and specific management is required, which is discussed in this review.
\end{abstract}

Key words: facial psoriasis, genital psoriasis, palmoplantar psoriasis, scalp psoriasis, special localization.

\section{Introduction}

Psoriasis is a chronic inflammatory dermatosis affecting $1-3 \%$ of the general population [1]. It is estimated that in about $70-80 \%$ of patients psoriatic skin lesions are mild and require only topical treatment. In the remaining group the severity of lesions is more pronounced and it is necessary to introduce more advanced therapeutic methods [2]. The most common areas of the body affected by the lesions include the elbows, knees, scalp, umbilicus, and lumbar region. Less frequently, psoriatic lesions can occur on the nails (23-27\%), face (49\%), palms and soles (12-16\%), or in intertriginous regions (21-30\%).

Patients with psoriasis represent a heterogeneous population with individual disease expression - different degrees and severity of skin involvement. It is commonly known that a significant physical, psychological, social and economic burden, and stigmatization are strongly associated with the disease. The decrease of the quality of life in psoriatic patients is connected with not only the body surface area affected but also with the special localization of the lesions. Thus, the special sites of psoriasis need appropriate management, which is discussed in this review [3].

\section{Scalp psoriasis}

The estimated incidence of scalp psoriasis among psoriatic patients is $45-56 \%$ of [3]. In one epidemiological study it was found that up to $90 \%$ of psoriatic patients may have the scalp affected by the disease at some point during their lifetimes [4, 5].

Scalp psoriasis manifests as erythematous patches and silvery white scales that flake (Fig. 1). The involved skin is exceedingly dry, often causing cracking and bleeding on its surface. The lesions on the scalp are mainly very pruritic. Many patients also complain about sensations of pain or burning. Patients with scalp psoriasis are particularly susceptible to the Koebner phenomenon - reformation of psoriasis as a result of injury including routine hair care. Scraping and scale removal can be a cause of secondary and temporary alopecia.

Involvement of the scalp in psoriatic patients has a significant negative impact on their quality of life. More than $70 \%$ of these patients report difficulty with

\section{Address for correspondence:}

Klaudia Dopytalska, Department of Dermatology, Centre of Postgraduate Medical Education, Central Clinical Hospital of the Ministry of the Interior, 137 Wołoska St., 02-507 Warsaw, e-mail: klaudia.dopytalska@cskmswia.pl

Submitted: 4.11.2018; Accepted: 5.12.2018 


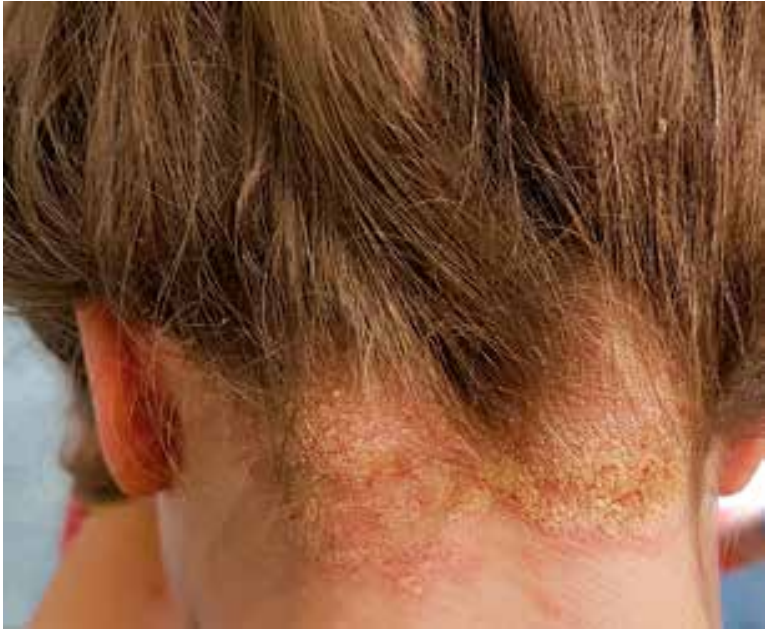

Fig. 1. Scalp psoriasis.

everyday activities. Patients often experience the feeling of shame, embarrassment or self-consciousness about their scalp lesions and as a consequence wear hats or grow their hair long to hide the defect $[4,6]$

A large group of psoriatic patients with severe scalp involvement present mild involvement of the body skin and in consequence they may not meet the criteria to receive systemic therapy for moderate-to-severe psoriasis vulgaris. Thus, the most frequent therapy is based on topical agents, such as coal tar, corticosteroids and vitamin D analogs. Nevertheless, it is challenging to apply them on the scalp due to hair - many patients quit this therapy as it is cosmetically unacceptable for them. These facts contribute to nonadherence and dissatisfaction with this therapeutic option. Additionally, topical agents usually have only a temporary effect and lead to frequent recurrence.

Localized ultraviolet B therapy (fiber-optic hair brushes) is a helpful alternative for patients, but devices are expensive and not widely available in medical facilities. It is also a time-consuming method and not every full-time working patient can afford it. The use of systemic agents, including methotrexate, cyclosporin A and biologics, is highly desirable in severe scalp psoriasis but currently still remains an off-label indication.

\section{Facial psoriasis}

About $20 \%$ of patients with psoriasis vulgaris have facial manifestation of the disease. Facial psoriasis is more often observed in patients with longer disease duration, family history of psoriasis and more severe psoriasis [7]. The most frequently involved areas are the upper forehead (76\%), lower forehead (52\%), periauricular area (46\%), ears (39\%), and cheeks (39\%). The nasolabial folds, eyelids and perioral area are rare sites of

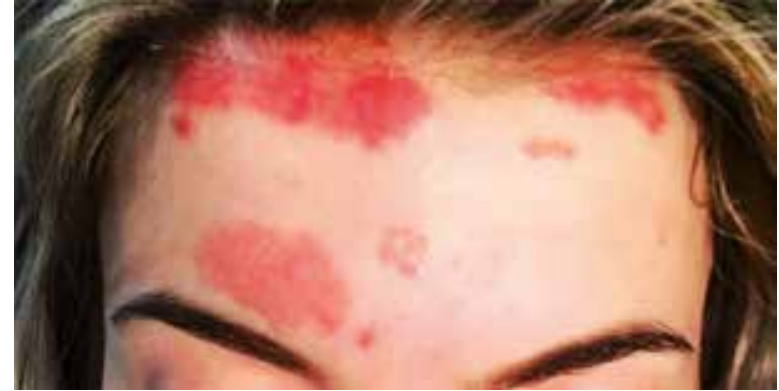

Fig. 2. True facial psoriasis.

psoriasis. It is proven that the vast majority of patients with facial psoriasis also have the scalp involved [4, 8].

Facial psoriasis includes three manifestations: sebopsoriasis, hair-line psoriasis and true facial psoriasis (Fig. 2). The first one can be considered as part of scalp psoriasis. Sebo-psoriasis is localized primarily in the seborrhoeic areas - the eyebrows and nasolabial fold - and has only mild scaling and is less indurated in comparison to psoriasis vulgaris. The typical psoriatic lesions on the face are characterized by sharply demarcated erythemato-squamous plaques with a classical overall morphology of chronic plaque psoriasis. Patients complain mainly about itchiness, soreness and oversensitivity of skin [9].

As lesions of the face are difficult to hide, patients fear negative stereotypes and societal judgment, which can cause them to feel isolated, ashamed, depressed and may even be a reason for suicidal tendencies. The involvement of the face in psoriasis can extend widely to many aspects of daily life - problems with intimacy and unemployment [7].

It is proven that facial involvement in psoriasis is a marker of more severe disease. In patients with facial psoriasis PASI scores of the whole body are generally higher than in patients without lesions on the face. The Koebner phenomenon is also more frequently reported (70.2\% vs. $29.8 \%$ ) [8].

The short-term treatment of facial psoriasis includes mild topical steroids, although it has to be mentioned that these agents increase the risk of acne, atrophy and ocular side effects. Topical vitamin D analogs and immunomodulatory calcineurin inhibitors (pimecrolimus, tacrolimus) are effective steroid-avoiding agents. Research to date on facial psoriasis is very limited and more studies are needed [10].

\section{Intertriginous areas}

Inverse psoriasis, also called intertriginous psoriasis or skin-fold psoriasis, is an uncommon form of psoriasis, which typically occurs in flexural areas - the groin, axillae, umbilicus, intergluteal cleft and external genitalia. 




Fig. 3. Intertriginous psoriasis in intergluteal cleft.

The skin lesions are well demarcated, smooth, erythematous plaques, which are not as scaly and infiltrated as in classic plaque type psoriasis, with shiny appearance (Fig. 3 and Fig. 4).

Maceration and superficial ulceration are also often present in intertriginous areas. These lesions may cause itching, irritation, soreness and often lead to decreased sexual health. Due to the localization in flexural areas and favorable environment, fungal infections very often occur $[3,11]$.

The prevalence of intertriginous psoriasis varies in many studies - from 3.2\% to $7 \%$ in Chinese populations to $12 \%$ to $36 \%$ among European patients with psoriasis. The statistical data depend on whether genital psoriasis is considered to be part of inverse psoriasis or a separate entity. Accordingly, precise prevalence of inverse psoriasis is difficult to estimate [11-15]. Moreover, inverse psoriasis, especially genital psoriasis, is assumed to be underreported in clinical practice [16]. Intertriginous psoriasis most often presents in the groins. Skin lesions may appear in skin folds alone or in association with psoriasis at extensor sites [11, 13].

The approach to topical treatment of inverse psoriasis should take into consideration increased percutaneous absorption of steroid and alcohol in skin folds and greater potential for local adverse events such as atrophy, striae, and telangiectasia. The skin flexures are more sensitive to topical corticosteroids due to thinner skin in that area. According to the recommendation first line treatment includes low- to mid-potency topical steroids, vitamin $D_{3}$ analogues (calcipotriol) and topical immunomodulator agents (pimecrolimus and tacrolimus). Low- to mid-potency topical steroids are recommended as the first line therapy, for a short period of time, especially the first 2-4 weeks of treatment. These treatment have been proven as more effective than calcipotriol or topical immunomodulator agents (pimecro-

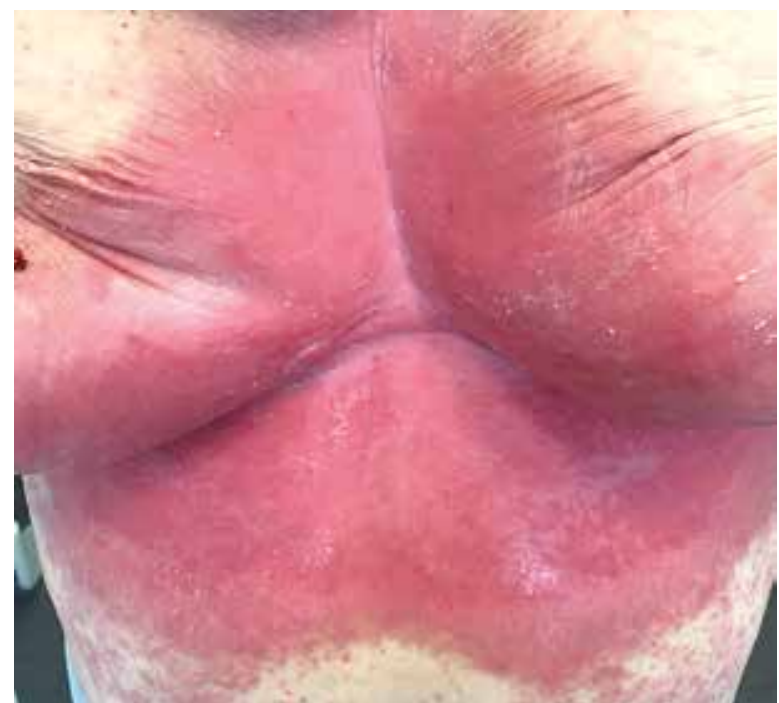

Fig. 4. Inverse psoriasis.

limus or tacrolimus). The efficiency of higher-potency topical steroid is also high, but increased frequency of adverse events has been reported. After improvement of psoriasis lesions, the frequency of using the topical steroid should be reduced or it should be finished. Subsequently after 2-4 weeks of topical steroids therapy, switching to calcipotriol or immunomodulator agents is recommended, which can be used with a low-potency topical steroid product one or two times per week for maintenance. However, in approximately 20\% of cases vitamin D analogues could cause irritation when used in skin folds. Therapy with corticosteroids requires special care and education of the patient for using the appropriate amount of topical products to reduce side effects.

Calcipotriol or one of the topical immunomodulating agents - pimecrolimus or tacrolimus - have been identified for long-term topical treatment for intertriginous psoriasis. The efficacy of these products in maintenance treatment has been proven. The advantages of analogs of vitamin D and topical immunomodulating agents include fewer local side effects compared to corticosteroids.

Second line recommended topical therapy includes emollients, tar-based products and antimicrobial agents. Even though antimicrobial therapy could be useful in treatment of skin folds due to increased microbial colonization, no formal studies have been performed [17-22].

Severe inverse psoriasis can be resistant to topical therapy. In these cases systemic treatment is required. Traditional oral systemic treatment (acitretin, methotrexate, cyclosporine), biologic agents and apremilast have been used with improvement, although further studies are essential. There are also reported cases in which dapsone has been used as an effective treatment option for intertriginous psoriasis [23]. 


\section{Genital psoriasis}

Genital skin involvement concerns approximately $63 \%$ of patients with psoriasis during the course of disease and up to $79 \%$ of patients with inverse psoriasis [24-26]. Furthermore, due to inadequate assessment and embarrassment of the patient, genital involvement remains often undiagnosed and untreated [24].

Genital psoriasis significantly affects quality of life and sexual health. Skin lesions in the genital area are characterized by intense redness and less scale and induration compared to plaque psoriasis (Fig. 5). The most frequent symptom reported by patients with genital psoriasis was genital itch. The data have shown that itching could appear in 82-100\% of patients with genital psoriasis. Less common, but bothersome symptoms are burning and pain $[16,27,28]$.

Genital involvement significantly influences sexual function. As a result of embarrassment patients with genital psoriasis often avoid sexual contacts, have less frequent intercourse and feel diminished sexual desire. Moreover, patients with genital involvement may feel pain during intercourse and exacerbation of symptoms after intercourse. In addition to the important impact on sexual health, genital psoriasis reduces the quality of life in general. Compared to patients without genital involvement, patients with genital skin lesions present decreased overall quality of life.

According to studies, genital psoriasis has an impact on physical activity, personal relationships, work and school, and emotions [16, 27-29].

Taking into account diminished life quality of patients with genital psoriasis, effective treatment is especially significant. Topical treatment of genital skin lesions is peculiarly challenging due to increased penetration of topical products, and sensitivity of the genital area, which could easily lead to sides effects. Low-potency topical corticosteroids are recommended treatment of genital psoriasis. Combination therapy with vitamin $D$ analogues or mild tar preparations should be considered [24, 26]. Some authors suggest short-term intermittent treatment with moderate-to-potent corticosteroids to induce skin resolutions, therefore shifting towards weaker topical steroids [26, 30, 31].

After topical corticosteroids, tar preparations are regarded as second-line topical therapy, which may be used independently or combined with topical steroids. Also, vitamin D analogues have proven to be effective in non-steroid topical treatment for genital skin lesions, but can cause irritation. In addition to tar preparations and vitamin D analogues, immunomodulator agents (pimecrolimus or tacrolimus) are considered to be second line therapy [24, 26, 32].
There are limited data about systemic treatment in isolated genital psoriasis. Resistant to topical therapy, severe cases may be treated with traditional systemic therapies, newer biologic therapies, or apremilast, although more studies about efficacy in the genital area are required. Clinical trials have shown the potential role of ixekizumab in patients with moderate-to-severe genital psoriasis [23, 32].

\section{Palmoplantar psoriasis}

Palmoplantar psoriasis (PP) accounts for about 12$16 \%$ of psoriasis cases. Palmoplantar psoriasis is often resistant even to strong local treatment; it should be classified as severe psoriasis. Patients with this disease report significant functional impairment and the occurrence of symptoms such as burning or pain, and worse health-related quality of life than in patients with other forms of psoriasis. Morphology may vary from thick, hyperkeratotic plaques with fissuring to pustular lesions [23, 33, 34].

Although palm and sole involvement is less than $5 \%$ of body surface area, patients may suffer from greater physical constraints than people with psoriasis localized in other areas [23].

The morphology of skin lesions allows one to distinguish between hyperkeratotic and pustular varieties.

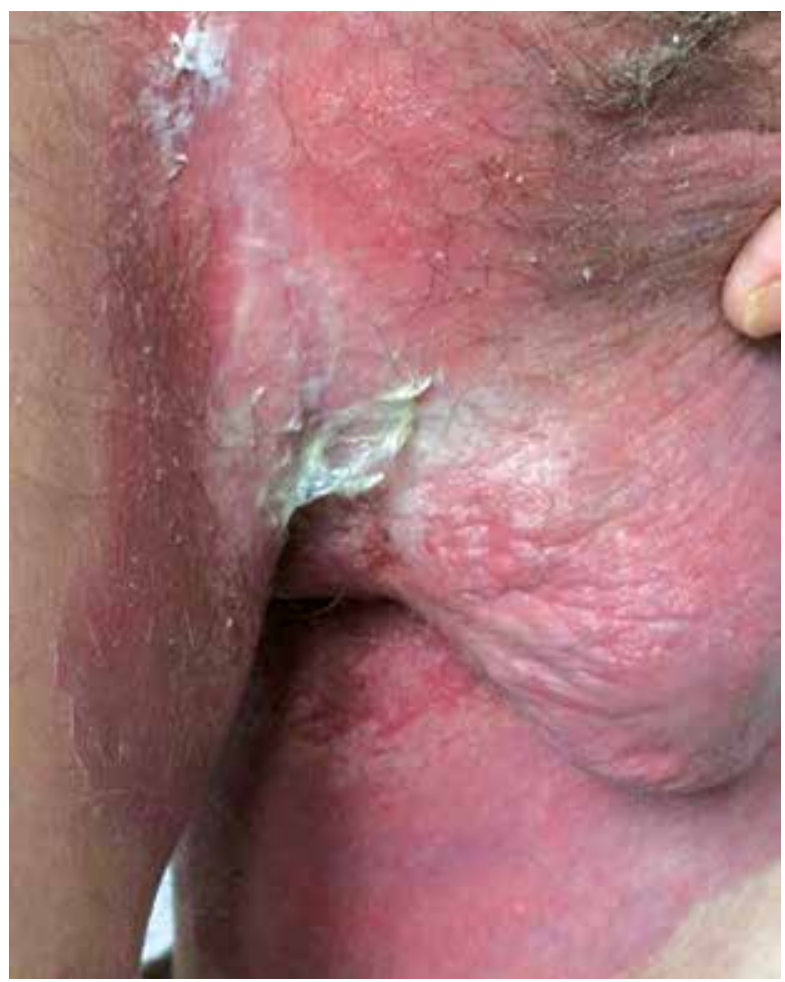

Fig. 5. Genital psoriasis. 
The former refers to sharply outlined, erythematous, scaly plaques with hyperkeratosis without the presence of pustular lesions. The pustular variety includes macroscopic, sterile pustules and erythema, with yellow-brown spots [1, 3, 34].

Diagnosis of psoriasis of palms and soles is not always straightforward, considering frequent clinical overlap with chronic eczema and frequent co-occurrence of these two conditions. Due to the fact that their treatment will vary, proper diagnosis is essential for a successful outcome $[1,3,19]$.

Despite the relatively small BSA, quality of life can be poor for patients with psoriasis of hands and feet due to pain and its visibility. Treatment should focus on alleviating pain as well as cosmetic improvement, as pain reduction and improvement in daily activities may be more important for patients than the total remission of skin lesions [23, 33-35].

The starting point is the use of strong local corticosteroids, which leads to short-term remission in about $1 / 5$ of patients. Occlusion of a corticosteroid under plastic or a hydrocolloid dressing increases the speed and degree of improvement. Other topical therapies include retinoids, calcipotriol, salicylic acid, vitamin D analogs and coal tar. However, chronic use of topical therapies does not always control changes, and long-term use of corticosteroids may cause unwanted side effects [34].

Phototherapy effects (including PUVA and NB-UVB) are visible in treatment, although data confirming the effectiveness of this method are limited. Moreover, multiple clinic visits for light treatment can be inconvenient for patients. Studies have shown the efficacy of excimer laser $(308 \mathrm{~nm}$ ) in a case series, but this is expensive and not widely available [19, 36-38].

Topical therapy and phototherapy are the first-line methods in the treatment of psoriasis of the hands and feet. However, about 2/3 cases of palmoplantar psoriasis are severe forms requiring general treatment using methotrexate, acitretin, cyclosporin A or newer biological therapies. The PDE4 inhibitor apremilast may be useful too. In refractory cases of pustular palmoplantar psoriasis, dapsone can be successfully used in a topical or oral form $[19,23,39]$.

Methotrexate (MTX) is a drug of choice in systemic treatment, due to its proven significant efficiency for palmoplantar psoriasis and severity index (m-PPPASI) scores. Acitretin is an alternative treatment, less effective than MTX [40].

Biological treatment might be considered when standard therapies fail. Many tumor necrosis factor inhibitors have shown variable efficacy. Infliximab failed to meet a primary endpoint of m-PPPASI 75 at week 14 compared with placebo. Newer biologic therapies such as secukinumab, ustekinumab and ixekizumab have significantly improved the efficacy of treating palmoplantar psoriasis [23, 41].

\section{Conclusions}

Psoriasis in special localizations significantly reduces quality of life. Moreover, some studies suggest that psoriatic lesions on the scalp, in the intergluteal cleft or perianal area could be a risk factor for development of psoriatic arthritis $[42,43]$. There is no consensus on the best therapeutic methods for the treatment of atypical forms of psoriasis, such as the scalp, face, intertriginous areas, genitals, hands, feet and nails. These areas are more resistant to treatment or too sensitive to be treated with strong topical drugs, resulting in more frequent use of systemic drugs $[1,19]$.

Systemic therapy, including biological therapy, is often indicated due to the strong influence on the quality of patients' life that psoriasis has. Despite the fact that only a small surface of above-mentioned localizations is usually affected by psoriasis, patients experience high levels of physical impairment and emotional distress. The problem with systemic therapy is that long-term effects of modern drugs have not yet been analyzed, due to the lack of appropriate data [23, 33].

Topical agents are another form of treatment of psoriasis. However, their application might be a problem especially in terms of tolerance, due to their toxicity. Due to a more targeted approach, new drugs may offer greater efficacy in management of psoriasis involving difficult-to-treat localizations compared to traditional systemic agents that are restricted to certain areas. The problem with systemic therapy is that the long-term effects of modern drugs have not yet been analyzed due to the lack of adequate data. The development of biological agents may result in faster and greater clearance of skin lesions than currently available therapies [1, 3, 19].

The authors declare no conflict of interest.

\section{References}

1. Michalek IM, Loring B, John SM. A systematic review of worldwide epidemiology of psoriasis. J Eur Acad Dermatol Venereol 2017; 31: 205-212.

2. Reich A, Adamski Z, Chodorowska G, et al. Psoriasis. Diagnostic and therapeutic recommendations of the Polish Dermatological Society. Part I: Mild psoriasis. Dermatol Rev/Przegl Dermatol 2018; 105: 225-243.

3. Merola JF, Li T, Li W, et al. Prevalence of psoriasis phenotypes among men and women in the USA. Clin Exp Dermatol 2016; 41: $486-489$ 
4. Aldredge LM, Higham RC. Manifestations and Management of Difficult-to-Treat Psoriasis. JDNA 2018; 10: 189-197.

5. Frez ML, Asawanonda P, Gunasekara C, et al. Recommendations for a patient-centered approach to the assessment and treatment of scalp psoriasis: A consensus statement from the Asia Scalp Psoriasis Study Group. J Dermatolog Treat 2014; 25: 38-45.

6. Sampogna F, Linder D, Piaserico S, et al. Quality of life assessment of patients with scalp dermatitis using the Italian version of the Scalpdex. Acta Derm Venereol 2014; 94: 411-414.

7. Alpsoy E, Polat M, Fettahlio Glu-Karaman B, et al. Internalized stigma in psoriasis: A multicenter study. J Dermatol 2017; 44: 885-891.

8. Woo SM, Choi JW, Yoon HS, et al. Classification of facial psoriasis based on the distributions of facial lesions. J Am Acad Dermatol 2008; 58: 959-963.

9. Canpolat F, Cemil BC, Eskioglu F, Akis HK. Is facial involvement a sign of severe psoriasis? Eur J Dermatol 2008; 18: 169-171.

10. Van de Kerkhof PCM, Murphy GM, Austad J, et al. Psoriasis of the face and flexures. J Dermatolog Treat 2007; 18: 351-360.

11. Omland SH, Gniadecki R. Psoriasis inversa: A separate identity or a variant of psoriasis vulgaris? Clin Dermatol 2015; 33: 456-461.

12. Fan X, Yang S, Sun LD, et al. Comparison of clinical features of $\mathrm{HLA}-\mathrm{CW}^{*} 0602$-positive and -negative psoriasis patients in a Han Chinese population. Acta Derm Venereol 2007; 87: 335-340.

13. Wang G, Li C, Gao T, et al. Clinical analysis of 48 cases of inverse psoriasis: A hospital-based study. Eur J Dermatol 2005; 15: $176-178$.

14. Dubertret L, Mrowietz U, Ranki A, et al. European patient perspectives on the impact of psoriasis: The EUROPSO patient membership survey. Br J Dermatol 2006; 155: 729-736.

15. Fouere S, Adjadj L, Pawin H. How patients experience psoriasis: Results from a European survey. J Eur Acad Dermatol Venereol 2005; 3 (Suppl 19): 2-6.

16. Meeuwis KA, van de Kerkhof PC, Massuger LF, et al. Patients' experience of psoriasis in the genital area. Dermatology 2012; 224: 271-276.

17. Wozel G. Psoriasis treatment in difficult locations: scalp, nails, and intertriginous areas. Clin Dermatol 2008; 26: 448459.

18. Khosravi H, Siegel MP, Van Voorhees AS, Merola JF. Treatment of Inverse/Intertriginous Psoriasis: Updated Guidelines from the Medical Board of the National Psoriasis Foundation. J Drugs Dermatol 2017; 16: 760-766.

19. Sarma N. Evidence and Suggested Therapeutic Approach in Psoriasis of Difficult-to-treat Areas: Palmoplantar Psoriasis, Nail Psoriasis, Scalp Psoriasis, and Intertriginous Psoriasis. Indian J Dermatol 2017; 62: 113-122.

20. Kreuter A, Sommer A, Huyn J, et al. 1\% Pimecrolimus, $0.005 \%$ calcipotriol, and $0.1 \%$ betamethasone in the treatment of intertriginous psoriasis. Arch Dermatol 2006; 142: 1138-1143.

21. Dubweb GA, Eldebani S, Alhaddar J. Calcipotriol cream in the treatment of flexural psoriasis. Int J Tissue React 2003; 4: 127-130.
22. Lebwohl M, Freeman AK, Chapman MS, et al. Tacrolimus ointment is effective for facial and intertriginous psoriasis. J Am Acad Dermatol 2004; 51: 723-730.

23. Merola JF, Qureshi A, Husni ME. Underdiagnosed and undertreated psoriasis: Nuances of treating psoriasis affecting the scalp, face, intertriginous areas, genitals, hands, feet, and nails. Dermatol Ther 2018; 31: e12589.

24. Beck KM, Yang EJ, Sanchez IM, Liao W. Treatment of Genital Psoriasis: A Systematic Review. Dermatol Ther (Heidelb) 2018; 8: 509-525.

25. Meeuwis KAP, Potts Bleakman A, van de Kerkhof PCM, et al. Prevalence of genital psoriasis in patients with psoriasis. J Dermatolog Treat 2018; 29: 754-760.

26. Meeuwis KA, de Hullu JA, Massuger LF, et al. Genital psoriasis: a systematic literature review on this hidden skin disease. Acta Derm Venereol 2011; 91: 5-11.

27. Yang EJ, Beck KM, Sanchez IM, et al. The impact of genital psoriasis on quality of life: a systematic review. Psoriasis (Auckl) 2018; 8: 41-47.

28. Cather JC, Ryan C, Meeuwis K, et al. Patients' perspectives on the impact of genital psoriasis: a qualitative study. Dermatol Ther 2017; 7: 447-461.

29. Ryan C, Sadlier M, de Vol E, et al. Genital psoriasis is associated with significant impairment in quality of life and sexual functioning. J Am Acad Dermatol 2015; 72: 978-983.

30. Welsh BM, Berzins KN, Cook KA, et al. Management of common vulval conditions. Med J Aust 2003; 178: 391-395.

31. Albert S, Neill S, Derrick EK, et al. Psoriasis associated with vulval scarring. Clin Exp Dermatol 2004; 29: 354-356.

32. Ryan C, Menter A, Guenther L, et al. Efficacy and safety of ixekizumab in a randomized, double-blinded, placebo-controlled phase IIIb study of patients with moderate-to-severe genital psoriasis. Br J Dermatol 2018; 179: 844-852.

33. Kragballe K. Management of Difficult to Treat Locations of Psoriasis Scalp, Face, Flexures, Palm/Soles and Nails. Curr Probl Dermatol 2009; 38: 160-171.

34. Sanchez IM, Sorenson E, Levin E, Liao W. The Efficacy of Biologic Therapy for the Management of Palmoplantar Psoriasis and Palmoplantar Pustulosis: A Systematic Review. Dermatol Ther (Heidelb) 2017; 7: 425-446.

35. Poulin Y, Crowley JJ, Langley RG, et al. Efficacy of adalimum$\mathrm{ab}$ across subgroups of patients with moderate-to-severe chronic plaque psoriasis of the hands and/or feet: post hoc analysis of REACH. J Eur Acad Dermatol Venereol 2013; 28: 882-890.

36. Carrascosa JM, Plana A, Ferrándiz C. Effectiveness and safety of psoralen-UVA (PUVA) topical therapy in palmoplantar psoriasis: a report on 48 patients. Actas Dermosifiliogr 2013; 104: 418-425.

37. Chen X, Yang M, Cheng Y, et al. Narrow-band ultraviolet B phototherapy versus broad-band ultraviolet $B$ or psoralen-ultraviolet A photochemotherapy for psoriasis. Cochrane Database Syst Rev 2013; (10): CD009481.

38. Goldberg DJ, Chwalek J, Hussain M. 308-nm Excimer laser treatment of palmoplantar psoriasis. J Cosmet Laser Ther 2011; 13: 47-49.

39. Bissonnette R, Pariser DM, Wasel NR, et al. Apremilast, an oral phosphodiesterase-4 inhibitor, in the treatment of pal- 
moplantar psoriasis: Results of a pooled analysis from phase II PSOR-005 and phase III Efficacy and Safety Trial Evaluating the Effects of Apremilast in Psoriasis (ESTEEM) clinical trials in patients with moderate to severe psoriasis. J Am Acad Dermatol 2016; 75: 99-105.

40. Janagond AB, Kanwar AJ, Handa S. Efficacy and safety of systemic methotrexate vs. acitretin in psoriasis patients with significant palmoplantar involvement: a prospective, randomized study. J Eur Acad Dermatol Venereol 2013; 27: e384-389.
41. Bissonnette R, Poulin Y, Guenther L, et al. Treatment of palmoplantar psoriasis with infliximab: a randomized, double-blind placebo-controlled study. J Eur Acad Dermatol Venereol 2011; 25: 1402-1408.

42. Busse K, Liao W. Which Psoriasis Patients Develop Psoriatic Arthritis? Psoriasis Forum 2010; 16: 17-25.

43. Patrizi A, Venturi M, Scorzoni R, et al. Nail dystrophies, scalp and intergluteal/perianal psoriatic lesions: risk factors for psoriatic arthritis in mild skin psoriasis? G Ital Dermatol Venereol 2014; 149: 177-184. 